\title{
A Survey on Mining Cryptocurrencies
}

\author{
Tejaswini Pawar a,1, Sagar Shirsat ${ }^{\mathrm{b}}$, Yaminee Patil ${ }^{\mathrm{c}}$, Dr. Vijay Sonawane ${ }^{\mathrm{a}}$, \\ Dhiraj Birari $^{\text {a }}$ \\ ${ }^{a}$ Department of Information Technology, MVPS's KBTCOE Nashik, INDIA \\ ${ }^{b}$ Senior Executive - Design, Crompton Greaves Ltd. Nashik, INDIA \\ ${ }^{c}$ Department of Information Technology, A P Shah Institute of Technology Thane, \\ INDIA
}

\begin{abstract}
Advanced monetary standards have acquired huge ubiquity nowadays. Bitcoin is the decentralized, disseminated, distributed virtual cash known cryptographic money. Bitcoin mining chips away at standard of the blockchain, which is believed to be one of this present century's sharp advancement. The blockchain is the arrangement of blocks that are associated so that in the current block there is the hash of the past block. Any adjustment of information in any block in a blockchain brings about a blunder in the entire blockchain. A strategy called mining, where excavators settle a complex numerical riddle, produces Bitcoins. The excavators contend as quickly as time permits to mine the Bitcoin and guarantee the award. Mining should be possible by a solitary individual or by a pool, where a lot of excavators join to mine a solitary block in an organization.
\end{abstract}

Keywords. Blockchain, Cryptocurrencies, Hash, Mining, Proof of Work (PoW)

\section{Introduction}

Cryptocurrency is digital asset powered by blockchain technology [1]. Cryptocurrency hold monetary value created by electricity and high- performance computer. It is form of digital money policed by millions of computers called miners on same network and created by mathematical computations. Cryptocurrency works through distributed ledger technology in decentralized manner. The entire cryptocurrency system collectively produces a decentralized cryptocurrency at a pace that is established when the system is generated and widely recognized. Hundreds of Cryptocurrencies, each with its own twist on blockchain technology and numerous intended uses, are available to buy or sell. Bitcoin was first distributed Cryptocurrency released as open-source software in 2009. Crypto currencies use a blockchain technology [2] which is basically a ledger containing a record ofall the transactions on it that have taken place.

The blockchain is decentralized, meaning that it is not hosted in one specific location and can thus not be compromised easily. The smallest unit of a blockchain is a block, and it is a holder containing all the data of the exchange. There are four fields to a block, or essential credits:

\footnotetext{
${ }^{1}$ Tejaswini Pawar, Assistant Professor, Department of Information Technology, MVPS's KBTCOE, India Email: pawar.tejaswini@kbtcoe.org.
} 
Previous hash: The estimation of the hash of the past block is put away by this trait and that is the means by which the blocks are associated with one another figure 1 .

1. Information: This is the accumulated arrangement of exchanges that were mined and approved and remembered for the block.

2. Nonce: The nonce is an arbitrary worth used to change the presentation of the hash esteem in a "proof of work" agreement calculation that Bitcoin employments. Hash esteem is planned to be created by each block, and the nonce is the boundary used to produce the hash esteem. The confirmation of work is the exchange check measure did in the blockchain.

3. Hash: This is the worth acquired through the going through the SHA-256 calculation of the past hash worth, information and nonce; it is the block's advanced mark.

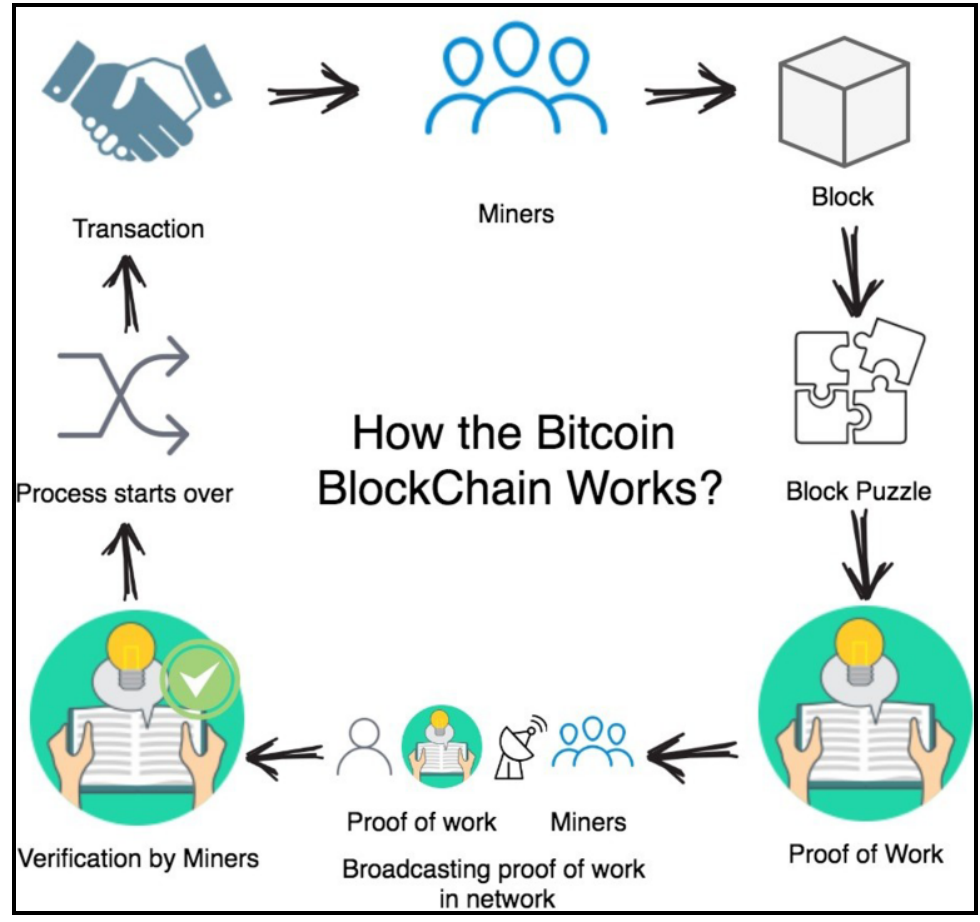

Figure 1. Working of Blockchain in Bitcoin

The means for running the organization incorporate the accompanying [3]:

- New exchanges are shipped off all nodes.

- Check if the exchanges are genuine.

- In a block, every node package new exchanges.

- Each node is attempting to track down a hard verification of-work.

- When a node finds a proof-of-work, the block is communicated to all nodes.

- The block is possibly affirmed by nodes if all exchanges in it are valid and not spent as of now. 
- Nodes pass on their endorsement of the block by working utilize the hash to make the following block in the chain, as the earlier hash of the affirmed block.

\section{Proof of Work (PoW)}

Proof of Work is consensus algorithm consist of complex cryptographic mathematical algorithm. Figure 2 it is introduced by Bitcoin to accumulate the amounts of cryptocurrency. It is verification process which contains complex computations. The value of nonce is hashed with SHA-256 and it will generate hash including number of zeros which is included in particular block in the chain. This high-level mathematical computation calculated by miners using high computing hardware. Bitcoin miners utilize the SHA-256 hashing calculation and determine the hash worth to deliver the hash. On the off chance that it is not exactly the given condition (the objective), at that point it is expected that the puzzle is tackled.

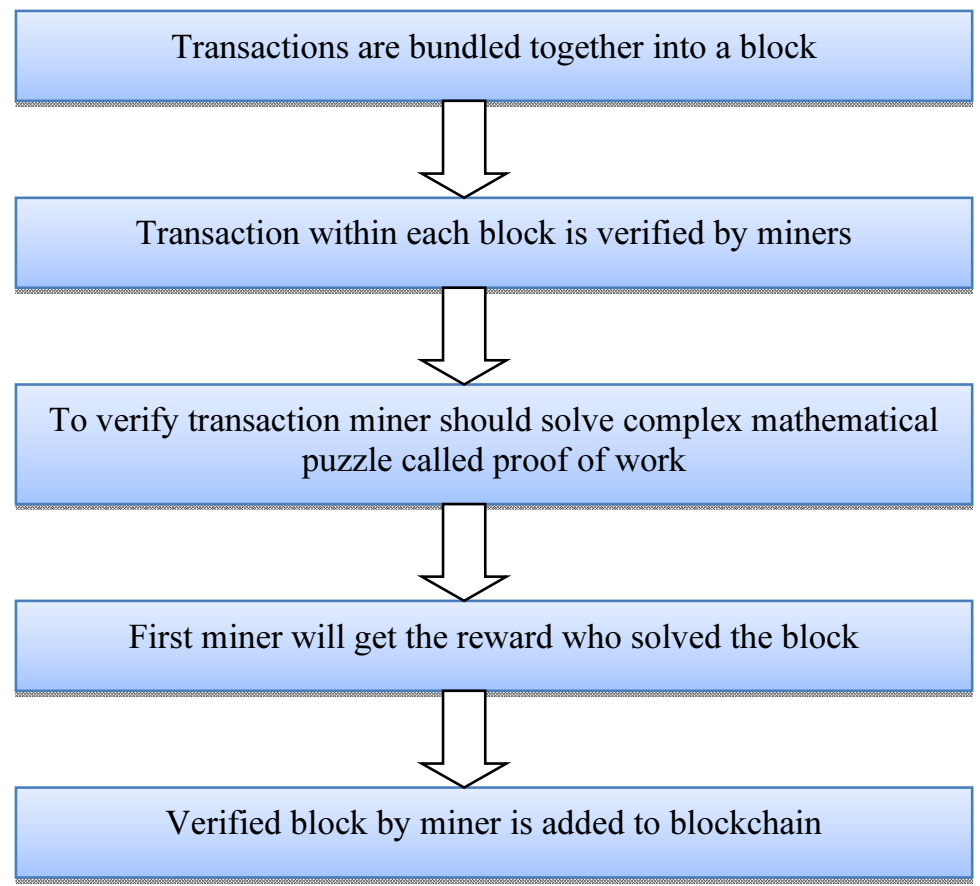

Figure 2. Flow of PoW

\section{Bitcoin Mining}

Mining is a distributed consensus mechanism which, by including them in the block chain, is used to validate pending transactions. It enforces a sequential order in the block chain, preserves the network's neutrality, and allows the state of the system to be agreed by various computers. Exchanges should be bundled in a block that consents to 
extremely severe cryptographic guidelines that will be checked by the organization to be approved. These guidelines forbid the adjustment of past blocks on the grounds that doing so would negate every single ensuing block. Mining additionally creates what might be compared to a serious lottery that keeps any person from progressively adding new blocks to the blockchain without any problem.

Mining guarantees that solitary substantial exchanges in the blockchain of some random digital money are affirmed. Mining is the technique for providing the organization of cryptographic money with a protected repayment instrument. Diggers are gadget proprietors who connect their Figureuring force and assets to the organization of digital money like Bitcoin dependent on "evidence of-work." A part of the cash that is mined as an award is acquired by the main excavator to approve another block for the blockchain. The Figure 3 shows interaction of how Bitcoin blockchain functions [3]. As the Bitcoin organization's hash rate developed, the general measure of 32-cycle nonce was exhausted excessively quickly. The additional nonce arrangement was acquainted with resolve this issue, whereby the coin base exchange is utilized as a wellspring of additional nonce to give a more extensive determination of nonce to be looked by the excavators. By using the following flowchart this method can be visualized:

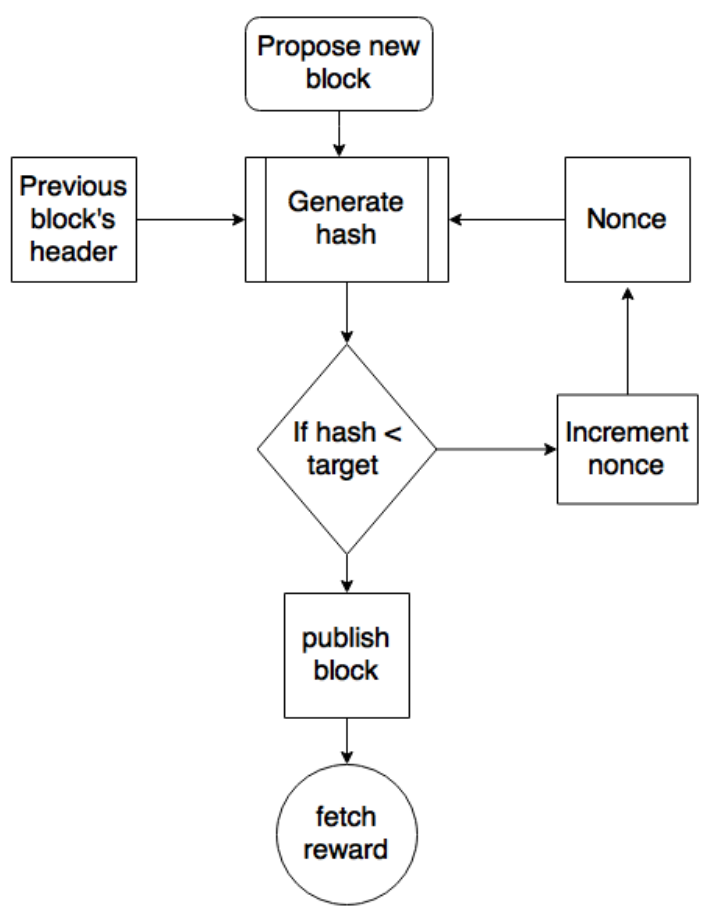

Figure 3. Flowchart of Mining

The calculation for mining comprises of the accompanying advances:

1. A header of the past block is recovered from the Bitcoin network.

2. Assemble a progression of organization exchanges into a block to be proposed. 
3. Using the SHA-256 calculation, process the twofold hash of the past block's header joined with a nonce and the recently recommended block.

4. Check that the subsequent hash is lower than the current (target) level of trouble; at that point, PoW is addressed. Because of the fruitful PoW, the block found is communicated to the organization and the award is gotten by excavators.

5. If the subsequent hash isn't not exactly the current degree of trouble (target), rehash the interaction in the wake of expanding the occasions.

\section{Hardware for Bitcoin Mining}

Bitcoin miners utilize their assets (equipment and power) to approve an exchange, and new Bitcoin are produced in the organization each time a block is mined. Following are the manners in which that portray utilization of various strategies for mining cryptographic money [5].

\subsection{CPU Mining}

Everything you need to use the CPU method to be able to mine is just a CPU and a couple of programs. Miners used standard processors to overcome the mathematical problems in the early days of Bitcoin, managing processor units (CPUs). It used to require some investment for mining Bitcoin and other cryptographic forms of money, despite the fact that the difficulty levels were less difficult than today. The degree of difficulty proceeds to change and grow, so the excavators have needed to build their preparing power too.

\subsection{Cloud Mining}

Cloud mining is likely the most famous route to mine digital currencies. Cloud mining has become so mainstream to a great extent since it gives individuals who might not have sufficient cash to purchase their hardware or who may essentially not be keen on claiming an equipment's the capacity to take an interest in the realm of digital currencies. Cloud mining is a technique wherein you pay a specific amount of cash to somebody (most ordinarily a huge organization) and "lease" their mining machine, called a "rig," and the mining cycle itself. This lease goes on for a settled upon span, in which all the income created by the apparatus are moved to your cryptographic money wallet (short the expense of power and upkeep). The people (organizations) that give these cloud mining administrations ordinarily have gigantic mining offices available to them with various ranches (ten or many apparatuses stacked and cooperating) and realize without a doubt how to mine cryptographic money.

There are two alternatives of cloud mining - free and charged. Numerous people searching for approaches to mine digital money would float towards the "free" decisions, however it has its hindrances (exceptionally sluggish mining speeds, additional conditions, and so on) Paying cloud mining ordinarily carries on like this:

A few hosts give you the choice to assemble and design your cloud mining plan. At that point look at the plans that the host gives and go through with the exchange (which 
means you pay the host), register digital currency wallet code and that is initial steps to mine

\subsection{GPU Mining}

The most mainstream and notable strategy for mining cryptographic forms of money is presumably GPU mining. GPU mining is likely the most widely recognized and notable interaction for mining digital forms of money. Designs cards are utilized by GPU apparatuses to mine cryptographic forms of money. A processor, a motherboard, cooling, rig outline and - obviously - a couple (2 - 8) illustrations cards are made of one single apparatus. A normal cost for a well-performing and pleasantly planned GPU mining rig will in general be around the $\$ 3000$ value range.

\subsection{ASIC Mining}

Miners use ASIC (application-explicit incorporated circuit) innovation, which was presented explicitly for mining Bitcoin and other digital forms of money. ASICs are very notable and regarded in light of the fact that they make insane amounts of cryptographic money contrasted with the GPU and CPU of their rivals. They burglarize different diggers who use GPU or CPU apparatuses of the capacity to stay aware of both hash paces and income. ASICS is so amazing. ASICs have additionally turned some remarkable cryptographic forms of money's economy - imagine if the bulk of profit will go to one miner with an ASIC ranch

\section{Conclusion}

Cryptographic forms of money are decentralized and run on the guideline of blockchain. Every one of the exchanges is reasonable and straightforward. The pace of trouble and the opposition between the excavators with the best accessible equipment makes mining more complicated. Digital currency mining needs a great deal of Figureuring and great equipment that can give a great hash rate with low energy. Miners should be cautious about picking equipment prior to beginning to mine digital money due to equipment costs. It is exceptionally high and the other extra expense during mining is the expense of power and fixes.

\section{References}

[1] Wikipedia" https:// en.wikipedia.org/ wiki/ History of bitcoin”. Accessed, 25 Jul. 2018

[2] https://www.simplilearn.com/bitcoin-mining- explained-article

[3] Suman Ghimire and Henry Selvaraj" A Survey on Bitcoin Cryptocurrency and its Mining". 26th International Conference on Systems Engineering (ICSEng), 18-20 Dec. 2018

[4] https://www.codeproject.com/Articles/125760 1/Introducing-the-Process-of-Mining-in-Blockchain

[5] https://www.bitdegree.org/crypto/tutorials/how-to-mine-cryptocurrency

[6] https://www.bitpanda.com/academy/en/lessons/what-is-bitcoin-mining-and-how-does- mining-work/

[7] https://bitcoin.org/en/how-it-worksA.N. Author, Article title, Journal Title 66 (1993), 856-890. 